\title{
Zur Abwehr gegen den Aufsatz von Dr. Schuster: „Ueber die Ausscheidung des Quecksilbers während und nach Quecksilberkuren"
}

ron

\author{
Dr. L. v. Vajda und Dr. H. Paschkis.
}

In der im letzten Hefte dieses Archivs erschienenen Arbeit "über die Ausscheidung des Quecksilbers vor und nach Quecksilberkuren" hat Herr Dr. Schuster die Ergebnisse unserer Quecksilberuntersuchungen, welche wir in der Schrift „über den Einfluss des Quecksilbers etc. . . ")" niedergelegt hatten, theilweise angezweifelt, theilweise als irrthümlich bezeichnet. Er gründet seine Zweifel einerseits auf die Unverlässlichkeit einer der angewandten Methoden, andererseits auf von ihm selbst erreichte Resultate.

Was die Unverlässlichkeit der Methode betrifft, so glauben wir nach der Erwiderung, welche dem Autor von seiten Prof. Ludwig's zu Theil geworden ist, kein Wort mehr darüber verlieren zu müssen. Wir hätten uns auch gewiss mit dieser realen, ausschliesslich die Ausführung der Methode betreffenden Richtigstellung begnügt, wenn der Autor nicht auch in anderer Beziehung Anlass zu ernstlichen Recriminationen gegeben hätte. Er kritisirt nämlich die Arbeit und beurtbeilt deren Resultate, ohne die erste auch nur gelesen zu haben. Dass dieser Vorwurf Herrn Dr. Schuster mit Recht trifft, geht schon daraus hervor, dass der Autor p. 52 sagt, „von beiden Autoren Vajda und Paschkis wurde die Ludwig'sehe Methode und demnach Zinkstaub zur Amalgamirung des zu suchenden Quecksilbers benützt", während wir p. 93 unserer Schrift ausdrücklich und wiederholt betonen, dass die ersteren 93 Fäle nach der Schneider'schen resp. nach der von Ludwig modificirten Schneider'schen Methode untersucht wurden. Da nun diese letztere Methode anf der Verwendung des elektrischen Stromes, nicht aber des Zinkstaubes, zur Ausscheidung des

) Ueber den Finfinss des Quecksilbers auf den Syphilisprocess v. Dr.

L. v. Vajda u. Dr. H. Paschkis. Wien, Braumüller 1880.

Vierteljahresschrift f. Dermaatol. u. Syph, 1882. 
Quecksilbers beruht, so erscheinen nicht nur der obige Ausspruch des Autors, sondern alle weiteren Schlussfolgerungen desselben aus der Luft gegriffen.

Hätto derselbe unsere Arbeit gelesen, so müsste er sich vor allem doch fragen, welche Fille eigentlich nach der ihm unerlässlich erscheinenden Ludwig'schen Methode untersucht worden seien. Dann hätte Dr. Schuster schon aus der Betrachtung der ersten 93 Fälle ersehen können, dass auch dort, wo der elektrische Strom statt des verdächtigen Zinkstaubes die Abscheidung des Quecksilbers besorgte, eine nachträgliche Ausscheidung bis 4 Monate und darüber nach der Einverleibung des Quecksilbers constatirt werden konnte (solche Fälle sind 3, 6, 17, 20, 107, 109).

Ohne uns anf weitere Details hier einlassen zu wollen, haben wir rücksichtlich der weitoren Einwendungen des Autors nur Folgendes zu bemerken: Es war uns sehr wohl bekannt, dass das Quecksilber auch auf anderen Wegen, als durch den Harn aus dem Körper zur Ausscheidung gelange und wir haben dieser Anschauung an vielen Stellen unserer citirten Abhandlung deutlichen Ausdruck gegeben.

Aus diesem Grunde glaubten wir von der Untersuchung der Fäces absehen zu können um so mehr, als wir ja in dem Auftreten des Qnecksilbers im Harn ein genügendes Kriterium für dessen Ausscheidung oder Remanenz vor uns hatten.

Wenn Herr Dr. Schuster weiters behauptet, dass er nach 6 Monaten kein Quecksilber im Harn und den Fäces gefunden habe, so mag dies für seine wenigen (6) Falle seine Richtigkeit haben, wenn er aber den Auspruch wagt: "Eine jahrelange Remanenz des Quecksilbers im Organismus ist demnach unwahrscheinlich", so geben wir ihm zu bedenken, dass es durchaus nicht angeht, auch nur gegen einen einzigen positiven Fall selbst tausende von negativen ins Feld zu führen.

Zum Schlusse wollen wir bezüglich der Ludwig'schen Mothode, deren Anwendung wir die Resultate unserer Arbeiten zum grossen Theile zu danken haben, Herrn Dr. Schuster nur fragen, wie or, da immer derselbe Zinkstaub bei den übrigen 103 Fällen in Verwendung gekommen ist, die häufigen negativen Resultate zu erklären in der Lage ist. 